\title{
Evaluation of Volume Expansion Therapeutic Effect in Acute Ischemic Stroke
}

\author{
Xiaodong Liu ${ }^{\mathrm{a}}$, Xinyu Du ${ }^{\mathrm{a}}$, Ran $\mathrm{An}^{\mathrm{a}}$, Xiaoling Wanga, \\ Guangjian $\mathrm{Liu}^{\mathrm{a}}$, Yi Bao,
}

\begin{abstract}
Background: Volume expansion therapy is widely used in clinical practice, whether expansion therapy has a certain effect on the recovery of neurological function in patients with ischemic stroke is very worth exploring. Our aim is to explore the value of dextran injection in patients with acute ischemic stroke.
\end{abstract}

Methods: A total of 101 patients with cerebral infarction were divided into two groups: the expansion group and the control group. National Institutes of Health Stroke Scale (NIHSS) scores were assessed at the time of admission and discharge.

Results: There was no statistically significant difference in neurological function improvement between the treatment group and the control group $(\mathrm{P}>0.05)$. Moreover, some factors including age, sex, NIHSS baseline score, systolic blood pressure before treatment, hypertension, diabetes mellitus, coronary heart disease, degree of cerebral arterial stenosis and option of treatment method have no statistically significant contribution to the patients $(\mathrm{P}>$ $0.05)$.

Conclusions: Dextran injection may not improve the degree of neurological deficit in acute ischemic stroke patients.

Keywords: Volume expansion therapy; Ischemic stroke; Dextran; NIHSS scores

\section{Introduction}

In the early stage of acute ischemic stroke, increased blood volume can improve cerebral blood flow and possibly increase oxygen exchange in brain tissue [1-2]. This physi-

Manuscript submitted May 31, 2019, accepted June 28, 2019

a Department of Neurology, Taihe Hospital Affiliated to Hubei University of Medicine, Shiyan City, Hubei Province, China

${ }^{b}$ Corresponding Author: Yi Bao, Department of Neurology, Taihe Hospital Affiliated to Hubei University of Medicine, Shiyan City, Hubei Province, China. Email:karlbaoyi@163.com

doi: https://doi.org/10.14740/jnr540 ological effect may be more pronounced in patients with hypovolemia. Moreover, some clinical observations indicate that hypovolemia is common in patients with early ischemic stroke [3-5]. In the past, researchers always hoped that hemodilution could increase patients' blood volume, reduce blood viscosity and increase the oxygen carrying capacity of brain tissue, so a large number of clinical trials were carried out. These clinical randomized trials selected albumin, dextran, hydroxyethyl starch, etc. as expansion drugs, while the control group used conventional treatment or crystalloid. However, these clinical trials have not yet reached a satisfactory conclusion [6-8].

Nevertheless, this unconventional treatment is still widely used in clinical practice. Therefore, whether expansion therapy has a certain effect on the recovery of neurological function in patients with ischemic stroke is very worth exploring. The purpose of this study is to further explore whether the treatment has potential application value by evaluating the therapeutic effect of expansion therapy in the acute phase of ischemic stroke.

\section{Materials and Methods}

\section{Subjects}

Retrospective analysis of 101 patients with cerebral infarction who were admitted to the Department of Neurology, Taihe Hospital, Shiyan City, Hubei Province from August 2018 to May 2019, including 57 males and 44 females, with an average age of $(61.1 \pm 12.2)$ years old.

The diagnosis complies with the 2018 version of the Chinese Acute Ischemic Stroke Diagnosis and Treatment Guideline issued by the Chinese Medical Association Neurology Branch Cerebrovascular Disease Group. Inclusion criteria are clinical manifestations of acute ischemic stroke without thrombolytic therapy. Exclusion criteria include patients with heart failure, liver and kidney dysfunction, pulmonary edema, hyponatremia and other diseases.

There were 52 cases in the expansion treatment group and 49 cases in the control group. There were no significant differences in gender and age between the two groups of patients with cerebral infarction at the time of enrollment.

This is a retrospective analysis. According to the local law no ethical approval was needed for such an investigation 
Table 1. Summary of Clinical Data of Patients

\begin{tabular}{|c|c|c|}
\hline & Expansion group $(n=52)$ & Control group $(n=49)$ \\
\hline Age $( \pm \mathrm{SD})$ & $58.9 \pm 10.8$ & $64.5 \pm 13.1$ \\
\hline Number of women (n, \%) & $18(34.6 \%)$ & $26(53.1 \%)$ \\
\hline Hypertension & $41(78.8 \%)$ & $46(93.9 \%)$ \\
\hline Diabetes & $12(23.1 \%)$ & $13(26.5 \%)$ \\
\hline Atrial fibrillation & $1(1.9 \%)$ & $1(2.0 \%)$ \\
\hline Other & $1(1.9 \%)$ & $2(4.1 \%)$ \\
\hline Systolic blood pressure at admission $( \pm \mathrm{SD})$ & $155 \pm 22$ & $153 \pm 23$ \\
\hline Systolic pressure at discharge $( \pm$ SD) & $137 \pm 18$ & $139 \pm 16$ \\
\hline NIHSS baseline score (M, R) & $5(3-8)$ & $3(2-6)$ \\
\hline
\end{tabular}

SD: standard deviation; n: number of cases, M: median; R: range. No significant difference in these clinical data $(P>0.05)$.

\section{Methods}

\section{Assessment of vascular stenosis}

All patients underwent transcranial Doppler color ultrasound (TCD) and digital subtraction angiography (DSA) to determine the degree of cerebral vascular stenosis. According to the degree of stenosis, the stenosis was divided into non-stenosis, mild to moderate stenosis $(<70 \%)$ and severe stenosis $(\geq$ $70 \%$ )/occlusion.

\section{National Institutes of Health Stroke Scale (NIHSS) score}

Patients' NIHSS scores were assessed on admission and discharge, respectively. The specific scoring method of the scale: each patient with acute ischemic stroke was scored according to the scoring rules by two attending physicians who were familiar with the NIHSS, the final result was the average of the two.

\section{Treatment methods and grouping}

The patients in the control group were treated with standard acute ischemic stroke treatment methods, namely anti-platelet aggregation (aspirin), lipid regulation (rosuvastatin) and free radical scavenger (edaravone). The patients in the expansion treatment group were expanded on the basis of the above, used low-molecular dextran injection $250 \mathrm{~mL}$ intravenous infusion once a day for more than 1 week.

\section{Statistical processing}

SPSS 22.0 software was used for data statistics. The count data were expressed as absolute numbers, and the comparison between groups was performed by $\chi^{2}$ test. Measurement data were expressed as ( \pm standard deviation (SD)), and independent data $t$-tests were used for comparison between clinical data groups. In addition, we performed a multivariate logistic regression analysis of the overall data. The test level is $\alpha=0.05$. The effects of various factors on NIHSS scores before and after treatment were evaluated by odds ratio (OR).

\section{Results}

\section{Summary of clinical data}

We collected the basic clinical information of patients in the expansion treatment group and the control group, including gender, age, past history, systolic blood pressure level at admission and discharge, NIHSS score at admission and discharge (Table 1). Through statistical analysis, we found no significant difference in these clinical data $(\mathrm{P}>0.05)$.

\section{Comparison of treatment effect between the two groups}

By comparing the improvement in NIHSS scores at the time of discharge between the patients in the treatment group and the control group (Table 2), we found that there was no significant difference between the two groups. $\left(\chi^{2}=1.703, \mathrm{P}=\right.$ 0.192).

\section{Evaluation of factors affecting the improvement of NIHSS score before and after treatment}

Because there was no significant difference in NIHSS score 
Table 2. Comparison of Treatment Effects Between the Expansion Group and the Control Group

\begin{tabular}{|c|c|c|c|}
\hline & Expansion group (n, \%) & Control group (n, \%) & Total (n\%) \\
\hline NIHSS score improvement group & $41(78.8 \%)$ & $33(67.3 \%)$ & $74(73.3 \%)$ \\
\hline NIHSS score no improvement group & $11(21.2 \%)$ & $16(32.7 \%)$ & $27(36.5 \%)$ \\
\hline
\end{tabular}

improvement between the two groups at discharge, we also used multivariate logistic regression analysis to further explore whether influencing factors including age, gender, NIHSS baseline score, pre-treatment systolic blood pressure, hypertension, diabetes, coronary heart disease, cerebral artery stenosis degree and the influence factors of treatment, etc. have a positive effect on the improvement of NIHSS scores in all patients. The results showed that the influencing factors were no significant statistical significance (Table 3).

\section{Discussion}

Based on the above results, we have not found that expansion therapy has a positive effect on the neurological function recovery of patients with acute ischemic stroke, which is consistent with most of the current studies [3-5], although theoretically, expansion therapy may improve blood perfusion in the ischemic area of patients with acute ischemic stroke and this view has been supported by some clinical or basic research [9-13]. However, as the relevant clinical trials continue to develop, we are getting more of the opposite conclusions. For example, as early as 1998, Aichner FT et al [14], through a multi-center clinical trial in Australia, believed that although expansion therapy was safe, it had no advantages in improving neurological defects and long-term prognosis of patients with acute cerebral infarction. In another systematic review, Asplund $\mathrm{K}$ et al [15] concluded that hemodilution therapy had similar advantages and disadvantages for the treatment of ischemic stroke, and all the included studies could not confirm that it can improve survival and functional prognosis.

In the expansion treatment, in addition to the use of dextran injection, there is also the use of albumin, is there any expansion? The Acute Stroke Albumin Research Clinical Trial (ALIAS) is a large-scale multi-center clinical trial conducted internationally in recent years, although phase I clinical trials have yielded favorable conclusions [16], but in the subsequent phase II trials, researchers thought [17, 18] ischemic stroke patients with intravenous albumin treatment didn't improve patients' prognosis after 90 days, but also increased the risk of intracranial hemorrhage and pulmonary edema. Some people even thought that increasing the dose of the expansion agent in the early stage of acute ischemic stroke might be detrimental to the recovery of nerve function [19]. As for why the above results are produced, there is no clear reason for this. Some scholars believe that excessive hemodilution may lead to decreased hematocrit and thus affect its oxygen-carrying capacity [20-23]. In addition, expansion therapy may aggravate cerebral edema in the acute stage of ischemic stroke.

In addition, as this study is a retrospective analysis, there may be some differences among all the included patients in the time of onset, timing and duration of administration, treatment of other combined diseases, etc. which may have an indirect impact on the included data and its analysis results. In addition, although the degree of cerebral vascular stenosis was analyzed and compared in the multivariate logistic regression analysis of all patients, the pathogenesis, such as TOAST type and infarct area, was not further stratified. Therefore, these

Table 3. Evaluation of Factors Affecting the Improvement of NIHSS Score Before and After Treatment

\begin{tabular}{lll} 
Variable & OR (95\% CI) & P value \\
\hline Gender & $0.577(0.197,0.691)$ & 0.316 \\
Age & $0.616(0.203,0.866)$ & 0.392 \\
Pre-treatment NIHSS score & $0.136(0.014,0.294)$ & 0.083 \\
Pre-treatment systolic pressure & $1.468(0.402,5.354)$ & 0.561 \\
Hypertension & $0.638(0.131,3.109)$ & 0.578 \\
Diabetes & $0.816(0.249,2.676)$ & 0.737 \\
Coronary heart disease & $1.228(0.310,4.863)$ & 0.769 \\
Cerebral artery stenosis & & 0.469 \\
$\quad$ No stenosis or mild to moderate stenosis & & 0.220 \\
$\quad$ Severe stricture & $0.418(0.040,4.420)$ & 0.481 \\
\hline
\end{tabular}

OR: odds ratio; $\mathrm{Cl}$ : confidence interval. 
problems need to be solved in future studies.

\section{Conclusions}

In this study, it was considered that the application of expansion therapeutic drugs (low molecular dextran) on the basis of conventional drugs (including antiplatelet aggregation drugs, lipid-regulating drugs, brain protectants, etc.) in patients with acute ischemic stroke could not effectively improve the degree of neurological impairment. We expect that a large sample of prospective clinical trials will be conducted in the future to explore the potential value of expanded drugs in the treatment of ischemic stroke.

\section{Acknowledgments}

We are grateful to the medical staff who have treated those patients carefully.

\section{Financial Disclosure}

None to declare.

\section{Conflict of Interest}

The authors declare that there is no conflict of interest.

\section{Informed Consent}

Not applicable.

\section{Author Contributions}

Data collection: XDL, XYD, RA, YB; data analysis: XDL, $\mathrm{XYD}, \mathrm{YB}$; manuscript preparation: $\mathrm{XDL}, \mathrm{XYD}, \mathrm{YB}$; reviewing of manuscript: XLW, GJL, YB.

\section{References}

1. Rink C, Gnyawali S, Peterson L, Khanna S. Oxygeninducible glutamate oxaloacetate transaminase as protective switch transforming neurotoxic glutamate to metabolic fuel during acute ischemic stroke. Antioxid Redox Signal. 2011;14(10):1777-1785.

2. Lin W, Powers WJ. Oxygen metabolism in acute ischemic stroke. J Cereb Blood Flow Metab. 2018;38(9):14811499.

3. Rodriguez GJ, Cordina SM, Vazquez G, Suri MF, Kirmani JF, Ezzeddine MA, Qureshi AI. The hydration influence on the risk of stroke (THIRST) study. Neurocrit Care. 2009;10(2):187-194.
4. Bhalla A, Sankaralingam S, Dundas R, Swaminathan $\mathrm{R}$, Wolfe CD, Rudd AG. Influence of raised plasma osmolality on clinical outcome after acute stroke. Stroke. 2000;31(9):2043-2048.

5. Schrock JW, Glasenapp M, Drogell K. Elevated blood urea nitrogen/creatinine ratio is associated with poor outcome in patients with ischemic stroke. Clin Neurol Neurosurg. 2012;114(7):881-884.

6. Chang TS, Jensen MB. Hemodilution for acute ischemic stroke. Stroke. 2015;46(1):e4-e5.

7. Bielewicz J, Kurzepa J, Czekajska-Chehab E, Kamieniak P, Daniluk B, Bartosik-Psujek H, Rejdak K. Worse neurological state during acute ischemic stroke is associated with a decrease in serum albumin levels. J Mol Neurosci. 2016;58(4):493-496.

8. Lilla N, Rinne C, Weiland J, Linsenmann T, Ernestus RI, Westermaier T. Early administration of HypertonicHyperoncotic Hydroxyethyl Starch (HyperHES) improves cerebral blood flow and outcome after experimental subarachnoid hemorrhage in rats. World Neurosurg. 2018;116:e57-e65.

9. Vorstrup S, Andersen A, Juhler M, Brun B, Boysen G. Hemodilution increases cerebral blood flow in acute ischemic stroke. Stroke. 1989;20(7):884-889.

10. Koller M, Haenny P, Hess K, Weniger D, Zangger P. Adjusted hypervolemic hemodilution in acute ischemic stroke. Stroke. 1990;21(10):1429-1434.

11. Strand T. Evaluation of long-term outcome and safety after hemodilution therapy in acute ischemic stroke. Stroke. 1992;23(5):657-662.

12. Belayev L, Liu Y, Zhao W, Busto R, Ginsberg MD. Human albumin therapy of acute ischemic stroke: marked neuroprotective efficacy at moderate doses and with a broad therapeutic window. Stroke. 2001;32(2):553-560.

13. Woessner R, Stoll M, Treib J. [Stroke therapy. What is established, what is new?]. MMW Fortschr Med. 2002;144(26):29-34.

14. Aichner FT, Fazekas F, Brainin M, Polz W, Mamoli B, Zeiler K. Hypervolemic hemodilution in acute ischemic stroke: the Multicenter Austrian Hemodilution Stroke Trial (MAHST). Stroke. 1998;29(4):743-749.

15. Asplund K, Israelsson K, Schampi I. Haemodilution for acute ischaemic stroke. Cochrane Database Syst Rev. 2000;2:CD000103.

16. Hill MD, Martin RH, Palesch YY, Tamariz D, Waldman BD, Ryckborst KJ, Moy CS, et al. The Albumin in Acute Stroke Part 1 Trial: an exploratory efficacy analysis. Stroke. 2011;42(6):1621-1625.

17. Ginsberg MD, Palesch YY, Martin RH, Hill MD, Moy CS, Waldman BD, Yeatts SD, et al. The albumin in acute stroke (ALIAS) multicenter clinical trial: safety analysis of part 1 and rationale and design of part 2. Stroke. 2011;42(1):119-127.

18. Martin RH, Yeatts SD, Hill MD, Moy CS, Ginsberg MD, Palesch YY, Parts A, et al. ALIAS (Albumin in Acute Ischemic Stroke) Trials: Analysis of the Combined Data From Parts 1 and 2. Stroke. 2016;47(9):2355-2359.

19. Miller JB, Lewandowski C, Wira CR, Taylor A, Burmeister C, Welch R. Volume of Plasma Expansion 
and Functional Outcomes in Stroke. Neurocrit Care. 2017;26(2):191-195.

20. Kellert L, Martin E, Sykora M, Bauer H, Gussmann P, Diedler J, Herweh C, et al. Cerebral oxygen transport failure?: decreasing hemoglobin and hematocrit levels after ischemic stroke predict poor outcome and mortality: STroke: RelevAnt Impact of hemoGlobin, Hematocrit and Transfusion (STRAIGHT) - an observational study. Stroke. 2011;42(10):2832-2837.

21. Sico JJ, Myers LJ, Fenton BJ, Concato J, Williams LS, Bravata DM. Association between admission haematocrit and mortality among men with acute ischaemic stroke. Stroke Vasc Neurol. 2018;3(3):160-168.

22. Fan AP, Khalil AA, Fiebach JB, Zaharchuk G, Villringer A, Villringer K, Gauthier CJ. Elevated brain oxygen extraction fraction measured by MRI susceptibility relates to perfusion status in acute ischemic stroke. J Cereb Blood Flow Metab. 2019:271678X19827944.

23. Guo D, Zhu Z, Zhong C, Peng H, Xu T, Wang A, Peng $\mathrm{Y}$, et al. Hemoglobin level and three-month clinical outcomes among ischemic stroke patients with elevated systolic blood pressure. J Neurol Sci. 2019;396:256-261. 